\title{
Sumsets of Sidon sets
}

\author{
by
}

IMRE Z. RuZsA (Budapest)

1. Introduction. A Sidon set is a set $A$ of integers with the property that all the sums $a+b, a, b \in A, a \leq b$ are distinct. A Sidon set $A \subset$ $[1, N]$ can have as many as $(1+o(1)) \sqrt{N}$ elements, hence $\sim N / 2$ sums. The distribution of these sums is far from arbitrary. Erdős, Sárközy and T. Sós $[1,2]$ established several properties of these sumsets. Among other things, in [2] they prove that $A+A$ cannot contain an interval longer than $C \sqrt{N}$, and give an example that $N^{1 / 3}$ is possible. In [1] they show that $A+A$ contains gaps longer than $c \log N$, while the maximal gap may be of size $O(\sqrt{N})$.

We improve these bounds. In Section 2, we give an example of $A+A$ containing an interval of length $c \sqrt{N}$; hence in this question the answer is known up to a constant factor. In Section 3 , we construct $A$ such that the maximal gap is $\ll N^{1 / 3}$. In Section 4 , we construct $A$ such that the maximal gap of $A+A$ is $O(\log N)$ in a subinterval of length $c N$.

2. Interval in the sumset. The constructions of Sections 2 and 3 are variants of Erdős and Turán's classical construction of a dense Sidon set (see e.g. [3]). We quote the common idea in the form of a lemma.

LEMma 2.1. If $p$ is a prime and $i, j, k, l$ are integers such that

$$
i+j \equiv k+l(\bmod p) \quad \text { and } i^{2}+j^{2} \equiv k^{2}+l^{2}(\bmod p),
$$

then either $i \equiv k$ and $j \equiv l$, or $i \equiv l$ and $j \equiv k$.

TheOREM 2.2. Let $c$ be a positive number, $c<1 / \sqrt{54}$. For sufficiently large $N$ there is a Sidon set $A \subset[1, N]$ of integers such that $A+A$ contains an interval of length $c \sqrt{N}$.

Proof. Let $p$ be the largest prime below $\sqrt{2 N / 3}-4$. For an integer $i$ let $a_{i}$ denote the smallest nonnegative residue of $i^{2}$ modulo $p$. Write $q=$ $2[p / 4]+1$. Let

Supported by Hungarian National Foundation for Scientific Research, Grant No. T 017433. 


$$
\begin{gathered}
s_{i}=2 i+q a_{i}, \quad t_{i}=N-i-q a_{i}, \\
A_{1}=\left\{s_{i}: p / 6<i<p / 3\right\}, \quad A_{2}=\left\{t_{i}: p / 6<i<p / 3\right\} .
\end{gathered}
$$

Our set will be $A=A_{1} \cup A_{2}$. Clearly $s_{i}+t_{i}=N+i \in A+A$, thus $A+A$ contains an interval of length

$$
[p / 3]-[p / 6]=p / 6+O(1) \sim \sqrt{N / 54} .
$$

It remains to show that $A$ is a Sidon set.

Suppose that $A$ contains four numbers that form a nontrivial solution of the equation $x+y=u+v$. These numbers can be distributed between $A_{1}$ and $A_{2}$ in five ways. Let Case $m, 0 \leq m \leq 4$, refer to the possibility that $m$ are in $A_{1}$ and $4-m$ in $A_{2}$.

Case 0 . This leads to the equation $s_{i}+s_{j}=s_{k}+s_{l}$, or

$$
2(i+j-k-l)=q\left(a_{k}+a_{l}-a_{i}-a_{j}\right) .
$$

Since $q$ is odd, we have

$$
q \mid i+j-k-l \text {. }
$$

These numbers satisfy

$$
(p+1) / 6 \leq i, j, k, l \leq(p-1) / 3,
$$

hence

$$
|i+j-k-l|<p / 3<q,
$$

thus (2.1) implies $i+j=k+l$, hence also $a_{i}+a_{j}=a_{k}+a_{l}$. This implies

$$
i^{2}+j^{2} \equiv k^{2}+l^{2}(\bmod p) .
$$

We conclude by Lemma 2.1 that $(i, j)$ is a permutation of $(k, l)$.

$\mathrm{Case} 1$. This leads to the equation $s_{i}+s_{j}=s_{k}+t_{l}$. Since $0<s_{i}<$ $p(q+1)$ and $t_{l}>N-p(q+1)$, the right side is always larger than the left, as

$$
3 p(q+1)<3 p \frac{p+4}{2}<N .
$$

Case 2. This means either $s_{i}+s_{j}=t_{k}+t_{l}$ or $s_{i}+t_{j}=s_{k}+t_{l}$. The first is clearly impossible, since the left side is smaller than the right. The second can be rewritten as

$$
2 i-2 k+l-j=q\left(a_{j}+a_{k}-a_{i}-a_{l}\right) .
$$

By (2.2) we have

$$
|2 i-2 k+l-j| \leq(p-3) / 3<q,
$$

thus we conclude that

$$
2(i-k)=l-j
$$


and

This equation implies

$$
a_{k}-a_{i}=a_{l}-a_{j}
$$

$$
k^{2}-i^{2}=(k-i)(k+i) \equiv l^{2}-j^{2}=(l-j)(l+j)(\bmod p) .
$$

By substituting $2(i-k)$ in place of $l-j$ this is transformed into

$$
(k-i)(2 l+2 j-k-i) \equiv 0(\bmod p) .
$$

By (2.2), the second factor satisfies $0<2 l+2 j-k-i<p$, thus it is not a multiple of $p$. Hence $k \equiv i$, which implies $k=i$ and we have a trivial solution.

Case 3 is treated like Case 1, and Case 4 like Case 0.

3. An ubiquitous sumset. We say that a set $X$ forms a d-chain in an interval if every subinterval of length $d$ contains at least one element of $X$.

Theorem 3.1. For all sufficiently large $N$ there is a Sidon set $A \subset[0, N]$ with the property that $A+A$ forms a $C N^{1 / 3}$-chain in the interval $[0,2 N]$. Here $C$ is an absolute constant.

Proof. Let $p$ be the smallest prime satisfying $2 p^{3}>3 N$. As before, we denote by $a_{i}$ the smallest nonnegative residue of $i^{2}$ modulo $p$. Our set will contain the numbers

$$
s_{i}=a_{i}+2 i p+2 b_{i} p^{2}, \quad 0 \leq i \leq p-1,
$$

with certain integers $b_{i}$.

First we show that these numbers form a Sidon set for an arbitrary choice of the integers $b_{i}$. Indeed, suppose that $s_{i}+s_{j}=s_{k}+s_{l}$, or

$$
a_{i}+a_{j}+2 p(i+j)+2 p^{2}\left(b_{i}+b_{j}\right)=a_{k}+a_{l}+2 p(k+l)+2 p^{2}\left(b_{k}+b_{l}\right) .
$$

By comparing the residues modulo $2 p$ we find that

$$
a_{i}+a_{j} \equiv a_{k}+a_{l}(\bmod 2 p) .
$$

Since the left and right sides are both in the interval $[0,2 p-2]$, this congruence implies equality. It also implies that

$$
i^{2}+j^{2} \equiv k^{2}+l^{2}(\bmod p) .
$$

Now we delete the $a$ 's from (3.1), divide by $p$ and find that

$$
i+j \equiv k+l(\bmod p) .
$$

From Lemma 2.1 we conclude that $(i, j)$ is a permutation of $(k, l)$.

Now we choose $b_{i}$ so that $A$ lies in $[0, N]$ and $A+A$ is dense in $[0,2 N]$. Certainly $s_{i} \geq 0$ if $b_{i} \geq 0$, and $s_{i} \leq N$ holds if we require that

$$
i+p b_{i} \leq \frac{N-p}{2 p} .
$$


Write

$$
M=\left[\frac{N}{2 p^{2}}\right]-1 .
$$

The largest value of $b_{i}$ that satisfies (3.2) is either $M$ or $M+1$; it is $M+1$ for

$$
i \leq i_{0}=\left[p\left\{\frac{N}{2 p^{2}}\right\}-\frac{1}{2}\right],
$$

and $M$ otherwise.

Observe that since $3 N \leq 2 p^{3}$, we have $3 M \leq p-1$.

We put $b_{3 r}=r$ for $0 \leq r \leq M, b_{3 r}=0$ for $M<r<p / 3, b_{3 r+1}=0$ for all $r$ and $b_{3 r+2}=M+1$ if $3 r+2 \leq i_{0}, b_{3 r+2}=M$ otherwise.

We have to show that the numbers $s_{i}+s_{j}$ appear in any interval of length $C N^{1 / 3}$. Since $0 \leq a_{i}<p=O\left(N^{1 / 3}\right)$, we have

$$
s_{i}+s_{j}=2 p\left(i+j+p\left(b_{i}+b_{j}\right)\right)+O\left(N^{1 / 3}\right),
$$

and it is sufficient to show that the numbers $i+j+p\left(b_{i}+b_{j}\right)$ form a $C$-chain in $[0, N / p]$ with a constant $C$.

Write

$$
\begin{aligned}
& B_{0}=\left\{a_{3 r}+p b_{3 r}: 0 \leq r \leq M\right\}, \\
& B_{1}=\left\{a_{3 r+1}+p b_{3 r+1}: 0 \leq r \leq(p-2) / 3\right\}, \\
& B_{2}=\left\{a_{3 r+2}+p b_{3 r+2}: 0 \leq r \leq(p-3) / 3\right\} .
\end{aligned}
$$

The elements of $B_{0}$ are the multiples of $p+3$ from 0 till $M(p+3)$. The elements of $B_{1}$ are the numbers $\equiv 1(\bmod 3)$ between 1 and $p-1$, so they form a 6 -chain in $[0, p+3]$. Hence $B_{0}+B_{1}$ forms a 6 -chain in the interval $[0,(M+1)(p+3)]$.

The elements of $B_{2}$ are the numbers

$$
2+p(M+1), 5+p(M+1), \ldots, 2+3 R+p(M+1),
$$

where $R$ is such that

$$
2+3 R+p(M+1) \leq \frac{N-p}{2 p}<2+3(R+1)+p(M+1),
$$

and after these the numbers

$$
2+3(R+1)+p M, \ldots, 2+3\left[\frac{p-3}{3}\right]+p M .
$$

The length of the gaps within a block is 3. By (3.5), the first element of the block in (3.6) is at most $N /(2 p)-p+3$, the difference between the last element of (3.6) and the first of (3.4) is at most 6, while the last element of (3.4) is at least $N /(2 p)-4$ again by (3.5). Hence $B_{2}$ forms a 6 -chain in $[N /(2 p)-(p+3), N /(2 p)]$. (One of the blocks may be empty; in this case we 
easily get the same conclusion.) Consequently, $B_{0}+B_{2}$ forms a 6 -chain in

$$
[N /(2 p)-(p+3), N / 2+M(p+3)] .
$$

By the definition of $M$ we see that

$$
N /(2 p)-(p+3)<(M+1)(p+3),
$$

thus the intervals overlap and $B_{0}+\left(B_{1} \cup B_{2}\right)$ forms a 12-chain in

$$
[0, N / 2+M(p+3)] \text {. }
$$

Finally, we consider $B_{2}+B_{2}$. It forms a 6 -chain in $[N / p-2(p+3), N / p]$ which overlaps with the previous interval, so together they form a 18-chain in $[0, N / p]$ as required.

4. With small gaps through a long interval. We show that if instead of the whole interval $[0,2 N]$ we are content with a positive portion, then the $N^{1 / 3}$ of the previous theorem can be reduced to $\log N$.

TheOREM 4.1. For all $c<1 / 5$ and sufficiently large $N$ there is a Sidon set $A \subset[0, N]$ with the property that $A+A$ forms a $C \log N$-chain in the interval $[N,(1+c) N]$. Here $C$ is a positive absolute constant.

The proof of this theorem is based on a different construction of a Sidon set, which we describe below.

Let $p$ be a prime, $g$ a primitive root modulo $p$ and write $q=p(p-1)$. For each $1 \leq i \leq p-1$ let $a_{i}$ denote the solution of the congruence

$$
a_{i} \equiv i(\bmod p-1), \quad a_{i} \equiv i(\bmod p), \quad 1 \leq a_{i} \leq q .
$$

The set $B=\left\{a_{i}\right\}$ forms a Sidon set modulo $q$, that is, the sums $a_{i}+a_{j}$ have all distinct residues modulo $q[4$, Theorem 4.4].

We need the following additional property of $B$.

Lemma 4.2. For a suitable choice of $g$ no interval of length $M=$ $\phi(p-1)^{1 / 3}$ contains more than two numbers whose residues modulo $q$ are elements of $B$.

Proof. All elements of $B$ satisfy $g^{b} \equiv b(\bmod p)$. Hence if there are three in an interval of length $M$, say $a, a+u, a+v$ with $0<u<v \leq M$, then the congruences

$$
g^{a} \equiv a, \quad g^{a+u} \equiv a+u, \quad g^{a+v} \equiv a+v(\bmod p)
$$

hold. On substituting the first into the others we obtain

$$
a\left(g^{u}-1\right) \equiv v, \quad a\left(g^{v}-1\right) \equiv u(\bmod p),
$$

hence (observe that $a \equiv g^{a} \not \equiv 0$ )

$$
u\left(g^{u}-1\right) \equiv v\left(g^{v}-1\right)(\bmod p) .
$$


For fixed $u, v$ this is an equation of degree $v$ in $g$, hence has at most $v$ solutions. By summing this for all pairs $u, v$ we conclude that there are less than $M^{3}$ values of $g$ for which such triplets exist. Since there are altogether $\phi(p-1)=M^{3}$ primitive roots, there must be a value of $g$ for which no such triplet exists.

Though it is likely that other dense Sidon sets, constructed via finite fields, also have a similar property, we were unable to establish it.

Pro of of The or em 4.1. Let $p$ be the largest prime satisfying $5 p(p-$ $1) \leq N$. We consider the set $B$ described above, with a $g$ as provided by Lemma 4.2 .

We divide $B$ into three subsets $B_{1}, B_{2}, B_{3}$ randomly, that is, all $3^{p-1}$ partitions are considered with equal probability. We put

$$
A=B_{1} \cup\left(B_{2}+q\right) \cup\left(5 q-B_{3}\right) \subset[1,5 q] \subset[1, N] .
$$

First we show that $A$ is a Sidon set for each partition. Suppose that $A$ contains four elements $x, y, u, v$ satisfying $x+y=u+v$. We call $B_{1} \cup\left(B_{2}+q\right)$ the lower half and $5 q-B_{3}$ the upper half of $A$.

If all four are from the lower half or all from the upper half, then this would violate the Sidon property of the residues modulo $q$.

If one is from the lower and three from the upper half, or three from the lower and one from the upper one, then we get a contradiction by comparing the magnitudes.

If two variables come from each half, then there are two possibilities. If $x, y$ are from one half and $u, v$ from the other, then again the magnitude of the sides leads to a contradiction. Assume finally that both sides contain a number from the lower and one from the upper half, say $x, u$ from the lower and $y, v$ from the upper. The residues of $x, u,-y,-v$ are elements of $A$ and they satisfy

$$
x+(-v) \equiv(-y)+u(\bmod q),
$$

which again contradicts the Sidon property of $A$ modulo $q$.

Now we begin to establish the chain property.

The numbers $a_{i}-a_{j}, i \neq j$, are all incongruent modulo $q$, and none of them is divisible by $p$ or $p-1$. Their number is $(p-1)(p-2)$, which is the same as the total number of residues modulo $q$ that are not divisible by $p$ or $p-1$. Hence for every $u$ such that $p \nmid u$ and $p-1 \nmid u$ there is exactly one pair $i, j$ such that

$$
a_{i}-a_{j} \equiv u(\bmod q) .
$$

In particular, if $1 \leq u \leq q$, then there is a pair $i, j$ such that

$$
a_{i}-a_{j}=u \quad \text { or } \quad a_{i}-a_{j}=u-q .
$$


If the first case holds, then we have

$$
5 q+u=a_{i}+\left(5 q-a_{j}\right)
$$

hence $5 q+u \in A+A$ if $a_{i} \in A_{1}$ and $a_{j} \in A_{3}$. In the second case we have

$$
5 q+u=\left(a_{i}+q\right)+\left(5 q-a_{j}\right),
$$

hence $5 q+u \in A+A$ if $a_{i} \in A_{2}$ and $a_{j} \in A_{3}$. In both cases

$$
\operatorname{Prob}(5 q+u \in A+A)=1 / 9 \text {. }
$$

Now take any interval $(s, s+t]$ of length $t=[C \log N]$ contained in $[5 q, 6 q]$. In this interval there may be at most one multiple of $p$ and one of $p-1$; each other has a chance $1 / 9$ of being in $A+A$. These events are not independent; we can claim independence only if the numbers $a_{i}, a_{j}$ used in the representations (4.1) are all distinct. For a fixed $n=5 q+u \in(s, s+t]$ we have to exclude those numbers that are in $a_{i}-B, a_{j}-B, B-a_{i}$ or $B-a_{j}$ modulo $q$. By Lemma 4.2 each of these sets has at most 2 elements in an interval of length $t<M$ (we have $M>p^{1 / 3-\varepsilon}$ by the familiar estimates for the $\phi$ function). Thus for any $n$ there are at most 8 other numbers that can spoil the independence. By the greedy algorithm we find $(t-2) / 9$ numbers in $(s, s+t]$, none divisible by $p$ or $p-1$, such that all the $a_{i}, a_{j}$ in their representations (4.1) are distinct. Hence the probability that none of them is in $A+A$ is less than $(8 / 9)^{(t-2) / 9}<1 / N$ if $C$ is large enough. Consequently, with positive probability this does not happen for any choice of $s$, which means that $A+A$ forms a $C \log N$-chain in $[5 q, 6 q] \supset[N,(6 / 5-\varepsilon) N]$.

Acknowledgements. This work was stimulated by conversations with Prof. P. Erdős.

\section{References}

[1] P. Erdős, A. Sárközy and V. T. Sós, On sum sets of Sidon sets I, J. Number Theory 47 (1994), 329-347.

[2] - - - - - On sum sets of Sidon sets II, Israel J. Math. 90 (1995), 221-234.

[3] H. Halberstam and K. F. Roth, Sequences, Clarendon, 1966.

[4] I. Z. Ruzsa, Solving a linear equation in a set of integers I, Acta Arith. 65 (1993), 259-282.

Mathematical Institute

Hungarian Academy of Sciences

Budapest, Pf. 127

H-1364 Hungary

E-mail: ruzsa@math-inst.hu 